\title{
LA (RE)LECTURE DU MYTHE CHEZ THÉSÉE D'ANDRÉ GIDE
}

Renata Lopes Araujo ${ }^{1}$

RÉSUMÉ: Cet article a pour but l'étude de l'une des nombreuses réécritures faites par André Gide, celle du mythe de Thésée. Dans ce livre Gide propose une manière différente de lire un texte bien connu et lui accorde aussi son interprétation personnelle, tout en invitant son lecteur à faire de même et pas seulement avec les mythes. Le lecteur gidien doit être préparé à faire partie activement de ce qu'il lit et à ne pas accepter un rôle passif.

MOTS-CLÉS: André Gide, mythe, Thésée, réécriture.

ABSTRACT: The goal of this article is to study one of the many rewritings made by André Gide, in this case the myth of Theseus. In this book Gide proposes a new form of read a well known text, and he also gives it a personal interpretation and invites the reader to do the same, and not only with the myths. Gide's reader must be prepared to be an active part of the text he reads and not to accept a passive role.

KEYWORDS: André Gide, myth, Theseus, rewriting. 

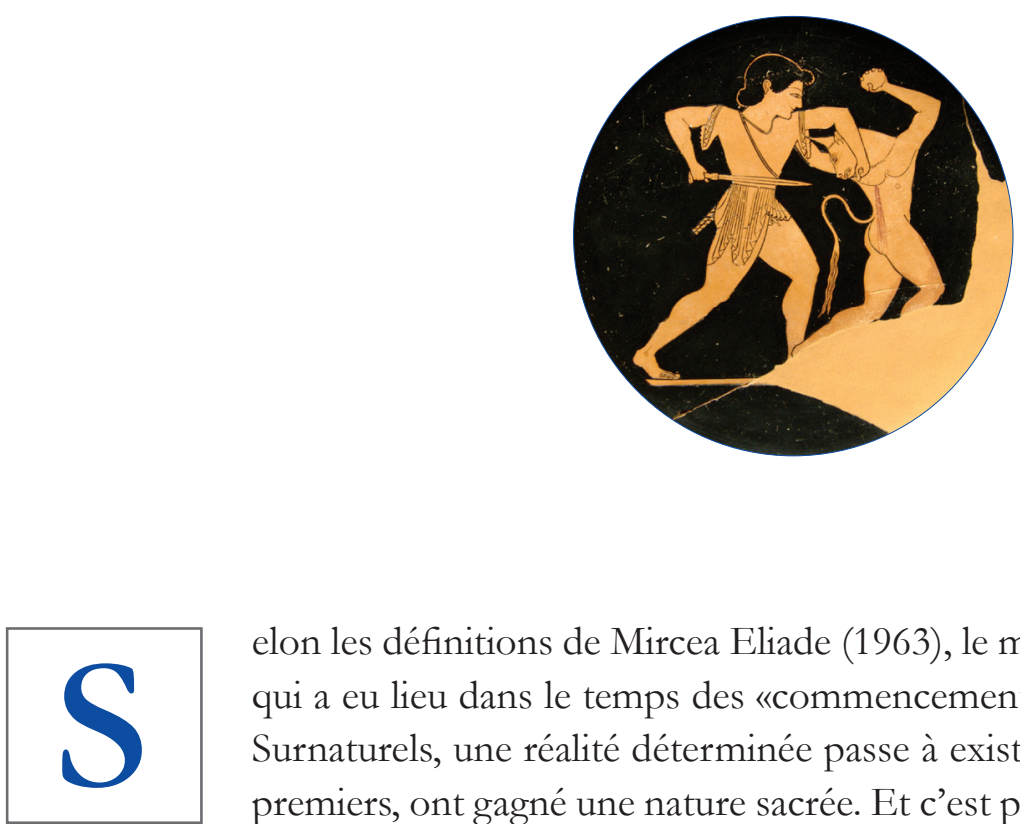

elon les définitions de Mircea Eliade (1963), le mythe raconte une histoire sacrée, et qui a eu lieu dans le temps des «commencements», un temps où, grâce à des Êtres Surnaturels, une réalité déterminée passe à exister. Les actes de ces Êtres, étant les premiers, ont gagné une nature sacrée. Et c'est par l'entrée du sacrée dans le Monde que celui-ci est devenu ce qu'il est, et que les hommes sont devenus ce qu'ils sont, c'est-à-dire des êtres culturels et mortels. Le mythe est, donc, considéré par les hommes primitifs comme une histoire vraie parce qu'il fait allusion à des choses qui existent réellement.

L'homme archaïque se croyait le résultat des événements mythiques - tout comme l'homme plus moderne s'est cru, pendant longtemps, le produit de l'Histoire. Toutefois, tandis que celui-ci ne sent pas le dévoir de connaittre toute l'Histoire qui précède sa naissance, l'homme primitif est non seulement obligé de se souvenir des mythes, mais il doit aussi en réactualiser la plupart. Donc, les mythes ne sont pas pour ces hommes que de simples histoires à ne pas oublier et à transmettre aux générations suivantes. Ce sont des réalités vivantes auxquelles il faut souvent revenir. Ils expriment une réalité éternelle, plus grande que celle où vivent ces hommes, et qui détermine leur vie, leurs activités et leurs destins ad aeternum.

Mais que se passe-t-il si, un jour, l'homme cesse de croire aux mythes? Cela est arrivé chez les Grecs et, d'après Eliade, à eux seuls. À ce moment-là, les mythes n'ont pas laissé d'exister tout de suite, mais ils ont commencé à être soumis à des analyses desquelles ils sortent «démythisés», dépourvus de leur caractère sacré et magique. Ils sont alors devenus de simples histoires fictives, prêtes à être relues et réinterprétées par les poètes.

Ce qu'il faut retenir est que le mythe donne la possibilité de créer quand on y croit et d'avoir un regard critique sur toute une conception de monde si l'on n'y croit plus. Dans les deux cas, on a la possibilité de le retravailler, soit pour créer à partir de lui, soit pour le mettre en question et, du coup, questionner aussi le monde dans lequel il fait partie.

Dans l'oeuvre d'André Gide, les mythes - et aussi les récits bibliques - ont une place importante. Dans un texte nommé Un esprit non prévenu, l'écrivain se plaint de l'«abandon» auquel les mythes ont été condamnés car, pour lui, ce sont des fables vivantes et qui nous concernent à 
tous. À chaque génération, leur substance se renouvelle et leur signification s'accroît: «Je prétends interroger la fable grecque d'une manière nouvelle, et vous dis que sa signification psychologique est intacte, qui c'est cette signification-là qui nous importe et qu'il appartient à notre époque de dégager» (GIDE, 1929, p. 73). Pour l'écrivain, il s'agit de textes qui contiennent l'essence éternelle de l'être humain et, en même temps, offrent la possibilité d'une réécriture dans laquelle Gide concentre les questions qui lui sont chères, telles que la liberté de l'homme, la sincérité envers soi-même et l'écriture en tant que moyen de connaissance personnelle. En général, il y a dans ses textes - et c'est le cas notamment pour Le Retour de l'enfant prodigue, Saul, Pbiloctète et Le Roi Candaule - une contraposition entre deux «morales ", l'une qui prône la soumission aux valeurs admises (plus spécifiquement les valeurs de la riche bourgeoisie, couche sociale à laquelle appartenait Gide) et l'autre qui défend la recherche d'un chemin dans lequel l'homme abandonne ces mêmes valeurs et crée une ligne de conduite personnelle malgré la pression venue non seulement du monde qui l'entoure mais surtout de sa propre conscience.

Nier les valeurs venues de l'extérieur signifie aussi rompre avec ce que l'on considère la vérité mais qui, pour Gide, n'est qu'un grand mensonge puisqu'il s'agit de règles créées avec l'objectif d'assujettir le naturel chez l'homme. En ce sens, les mythes et les histoires bibliques ont aussi une valeur révélatrice puisqu'à travers un récit dont le contenu est bien connu il est possible d'en faire ressortir une signification nouvelle, ce qui montre justement le caractère de construction de toute sorte de discours. Et en montrant au lecteur d'autres possibilités d'interprétation pour de textes dont le sens a été fixé pendant longtemps, Gide expose aussi le leurre auquel peut nous soumettre la littérature si nous la considérons seulement comme une chose figée et vers laquelle on se tourne afin de recevoir passivement un savoir. Ce savoir, il faut le construire à partir de la lecture mais de façon individuelle et, pour cela, l'auteur des Caves du Vatican exigeait de son lecteur une posture active et d'engagement envers ses textes; c'est ce que l'on peut constater dans l'avertissement de Paludes: «Un livre est toujours une collaboration, et tant plus le livre vaut-il, que plus la part du scribe y est petite (...). Attendons de partout la révélation des choses: du public, la révélation de nos oeuvres» (GIDE, 1920. p. 11).

Pour ce qui est de la lecture gidienne du mythe de Thésée, ce ne sera pas différent. L’auteur y inscrit, d'une part, sa propre vision de la littérature en tant que manifestation d'un parcours personnel, choisi sans l'interférence d'une morale contraignante - en tenant compte des implications parfois négatives d'un tel choix - et, d'autre part, réaffirme la liberté du lecteur à l'égard du texte, car Thésée n'est pas un narrateur des plus fiables, comme nous le verrons par la suite.

En ce qui concerne le mythe proprement dit, rappelons l'histoire en quelques lignes: les habitants d'Athènes devaient souvent livrer 7 garçons et 7 filles à Minos, roi de Crète, qui les donnaient à manger au Minotaure, un monstre qui était enfermé dans un labyrinthe construit par l'architecte Dédale. Décidé à livrer Athènes du joug du Minotaure, Thésée penètre dans le labyrinthe et pour ne pas s'y perdre, il portait une pelote de laine dont l'autre bout était tenu par Ariadne, fille du roi Minos. Après avoir tué le monstre, il part avec Ariadne et ses compagnons pour la Grèce. Mais à l'approche du port, il oublie de mettre les voiles blanches qui devaient prévenir son père de son retour et, quand Egée voit le navire sans les voiles en question, fou de douleur et de chagrin, il se jette dans la mer qui porte son nom. 
Chez les héros mythiques, Gide met en valeur ce qu'il appelle leur insouciance à l'égard des conventions. Selon lui, ils agissaient toujours d'après «le conseil de leur pente, de leur passion bonne ou mauvaise, de leur vertu, de leur orgueil ou de leur fantaisie, mais qu'il ne leur arrivait jamais de peser longuement (...) [le propos] que la raison reconnaitra pour préférable (...)» (GIDE, 1929, p. 85). A l'exception d'Hercule, les héros n’hésitent pas avant d'agir, et c'est ce que les rend intéressants aux yeux de Gide, pour qui l'homme doit vivre toujours selon son coeur, car la raison le contraint, l'empêche d'agir et d'être sincère envers soi-même. Son héros mythique agit motivé par son caractère, ses désirs et, dans le cas de Thésée, par la volonté de défi: il est toujours en quête d'une nouvelle aventure et, pour y arriver, il est prêt à laisser tomber tout le reste et à «passer outre».

La critique en général considère le Thésée comme une sorte de testament de Gide et un message de sagesse, spécialement parce que c'est son dernier texte de fiction, mais il me semble qu'il s'agit plutôt d'une contestation parodique de la parole littéraire pleine de sous-entendus, un peu comme une espèce de pastiche de l'écriture tragique. Cette contestation ferait «bouger» le mythe, c'est-à-dire, le renouvellerait et le réinterpréterait à la fois, sans pour autant modifier son essence.

Ce processus a lieu de plusieurs façons. Et un bon exemple est dans le récit d'un épisode important, la mort du père de Thésée. Dans le mythe, comme nous l'avons vu, cela est attribué à un oubli, « celui de remplacer par des voiles blanches les voiles noires du bateau qui me ramenait de Crète, ainsi qu'il était convenu si je revenais victorieux de mon entreprise hasardeuse » (GIDE, 1947, p. 17). Cependant, le Thésée gidien nous dit que son père, après avoir été rajeuni par Médée, obstruait «[sa] carrière, alors que c'est à chacun son tour» (GIDE, 1947, p. 18). Cela suggère donc que l'«oubli» n'a pas été si accidentel que cela, ce qui contribue beaucoup à nous faire questionner non seulement l'écriture du mythe, mais aussi l'image que nous avons d'un héros mythique.

Un autre exemple de la transformation "banalisante" du mythe se trouve dans le chapitre XI où il est question de l'abandon d'Ariane à Naxos:

Elle me poursuivait, me pourchassait, me traquait (...). L'on dit que, quelques temps après que nous l'y eûmes laissée, Dionysios vint l'y rejoindre et qu'il l'épousa; ce qui peut être une façon de dire qu'elle se consola dans le vin (...). Et l'on me permettra de remarquer que, sans mon abandon, ne fût advenu rien de tout cela, si avantageux pour elle (GIDE, 1947, p. 91-93).

L'enfer, pour lui, c'est toujours les autres et, en plus, il nous dit avoir fait une "bonne action" puisque la jeune femme est devenue connue grâce à lui...

Notons aussi, dans l'extrait ci-dessus et dans les suivants, la trivialité, voire vulgarité, des discours, non seulement en ce qui concerne ceux de Thésée mais aussi par rapport aux autres personnages, ce qui diffère complètement de ce qui, d'après Aristote, était convenable pour un mythe, c'est-à-dire, une tragédie, la « representação de uma ação nobre e completa, com uma certa extensão, em linguagem poetizada (...) que pela piedade e pelo terror opera a catarse desse gênero de emoções » (MALHADAS, 2003, p. 17). Cependant, selon Mikhail Bakhtin (1988), les tragédies 
grecques étaient d'habitude accompagnées par ses contreparties comiques, et celles-ci ne mettaient pas en question les mythes eux-mêmes mais leur représentation, et c'est exactement ce qui fait Gide, qui cherche justement à montrer la fausseté de cette représentation. Et cette banalité des discours des personnages n'en est qu'une preuve de plus. Voyons une des phrases d'Ariane: «Ce n'est que grâce à moi, que par moi, qu'en moi, que tu pourrais te retrouver toi-même. C'est à prendre ou à laisser. Si tu me laisses, malheur à toi. Donc commence d'abord par me prendre» (GIDE, 1947, p. 49).

Un autre élement remarquable est l'hyperbole lyrique qui, dans un récit où le langage est souvent familier, paraît complètement déplacée et ridicule: «Quelle terrasse! Et de quel palais! Ô jardins en extase, suspendus dans l'attente d'on ne savait quoi, sous la lune!» (GIDE, 1947, p. 47). Le lecteur avisé verra dans ces phrases dont les accents raciniens ne font doute à personne une preuve du mensonge auquel l'on peut se soumettre lorsqu'on regarde le texte littéraire comme un «mode d'emploi» pour le réel. En exposant quelques-unes des tournures compliquées dont la langue est capable pour représenter la simple vue d'un jardin, le narrateur met en relief justement le caractère artificiel de cette même représentation, puisqu'il lui serait tout à fait possible d'en parler en d'autres termes en exprimant les mêmes sensations - c'est d'ailleurs ce que l'on constate avec les propos banals des personnages mythologiques. Bref, il place l'illusion du réel véhiculé par la littérature dans la construction faite par le discours, c'est-à-dire dans la forme et pas nécessairement dans le fonds; le littéraire ne réproduit pas le réel mais donne l'impression de le faire par l'organisation qu'il imprime à une matière désordonnée telle que la vraie vie. Et si l'on prend une tragédie comme Phèdre et l'on compare son langage avec celui du morceau que nous venons de lire, on commence à regarder celui-là d'une manière plutôt critique:

Ah! le voici. Grands dieux! À ce noble mantient

Quel œil ne serait pas trompé comme le mien?

Faut-il que sur le front d'un profane adultère

Brille de la vertu le sacré caractère?

Et ne devrait-on pas à des signes certains

Reconnaître le cœur des perfides humains (RACINE, 2001, p. 63).

Par rapport à un autre aspect de la relecture gidienne - le discrédit total de l'action divine sur les humains et, par conséquent, la disparition de l'idée de soumission humaine aux puissances supérieures - l'extrait où Thésée raconte l'épisode des pierres précieuses est emblématique. Dans le mythe, elles les lui sont données par Poséidon, le dieu des mers. Mais notre héros nous raconte l'épisode autrement:

Comme une brise assez forte s'élevait, il advint qu'une longue écharpe fût enlevée des épaules d'Ariane. Le souffle la dirigea vers moi. (...) Aussitôt, me dépouillant du justacorps qui m'engonçait, je ceignis autour de mes reins cette écharpe, la passai entre mes cuisses et, et la ramenant par devant, l'assujettis. Il semblait que ce fût par pudeur (...); mais ce faisant je pus dissimuler la ceinture de cuir que je 
conservais, à quoi pendait une escarcelle. Dans celle-ci, je n'avais pas de piéces de métal, mais bien quelques pierres de prix (GIDE, 1947, p. 36-37).

Il ne compte pas sur les dieux: il faut donc faire appel aux moyens humains.

Chez Thésée il y a un grand questionnent à propos du discours, et non seulement par rapport à celui qui entoure les mythes, mais d'une façon générale: à qui le lecteur doit faire confiance, lorsque la narration en première personne est suspecte puisque subjective et la représentation est mensongère? Dans l'oeuvre de Gide, la littérature est présentée comme une monnaie fausse quand elle s'érige en transmetteuse de vérités, comme une copie conforme du réel; l'écrivain dénonce un échange malhonnête entre l'auteur et le lecteur. Le premier plait au public en général en ne lui offrant que ce que celui-ci souhaite récevoir et dans des formes convenues; le lecteur, en revanche, fait semblant de trouver dans la littérature l'image même de la vie. Et les narrateurs y sont pour beaucoup, surtout quand ils sont aussi les protagonnistes de leurs récits puisque c'est avec eux que le mensonge se propage. En utilisant un principe d'écriture qu'il avait identifié chez Dostoïevski, à savoir l'établissement d'une distance considérable entre les propos des personnages et les opinions de leur créateur - ce qui par ailleurs leur accorde une certaine liberté vis-à-vis de l'écrivain — Gide fait valoir les erreurs de jugement qu'ils commettent tout au long du récit et, par là, montre au lecteur qu'ils ne sont pas tout à fait fiables. Ils se présentent comme des narrateurs classiques, détenteurs d'un point de vue à toute épreuve et, de fil en aiguille, le lecteur soupçonne leur imposture à l'aide des actes et des paroles qui exposent la partialité de ce qu'ils disent. Avec Gide, il n'est plus possible d'envisager la littérature en tant qu'instrument d'accès à des explications sur la vie; il faut la prendre par ce qu'elle est, c'est-à-dire une représentation de cette même vie mais que lui accorde une organisation qu'elle ne possède pas, qui communique le réel mais ne le reproduit pas pour autant. Et c'est par le biais de narrateurs tels que Thésée que la fausseté d'une manière mimétique de regarder les textes est montrée en toute son étendue, puisqu'ils dévoilent la partialité de tout point de vue et, par là, amènent le lecteur à soupçonner toute sorte de récit qui essaie de lui faire croire à une vérité.

Dans Thésée, on a l'impression que l'on nous trompe, qu'il faut chercher la vérité qui se cache entre les lignes, dans ce qu'il y a de plus banal, comme nous montre la fin du livre, qui n'est qu'une collection de stéréotypes ayant pour but la construction d'une image qui, pendant tout le récit, nous a été montrée de la façon la plus ambiguë:

C'est consentant que j’approche la mort solitaire. J'ai goûté des biens de la terre. Il m'est doux de penser qu'après moi, grâce à moi, les hommes se reconnaîtront plus heureux, meilleurs et plus libres. Pour le bien de l'humanité future, j’ai fait mon oeuvre. J'ai vécu (GIDE, 1947, p. 114).

\section{RÉFÉRENCES BIBLIOGRAPHIQUES:}

BAKHTIN, M. Questões de Literatura e de Estética: A teoria do romance. São Paulo: Editora Unesp/ Hucitec, 1988. 
ELIADE, M. Aspects du mythe. Paris: Gallimard, 1963.

GIDE, A. Paludes. Paris: Gallimard, 1920.

. Un esprit non prévenu. Paris: Kra, 1929.

Thésée. Paris: Gallimard, 1947.

MALHADAS, D. Tragédia grega. São Paulo: Ateliê Editorial, 2003.

RACINE, J. Phèdre. Paris: Librio, 2001. 